\title{
Scanning Hard X-Ray Microscopy Based on Be CRLs
}

Andreas Schropp $^{1 *}$, Dennis Brückner ${ }^{1,2,3}$, Jessica Bulda ${ }^{1,2}$, Gerald Falkenberg ${ }^{1}$, Jan Garrevoet ${ }^{1}$, Frank Seiboth $^{1,4}$, Felix Wittwer ${ }^{1,2}$, Frieder Koch $^{5}$, Christian David ${ }^{5}$, and Christian G. Schroer ${ }^{1,2}$

${ }^{1}$ Deutsches Elektronen-Synchrotron (DESY), Notkestr. 85, D-22607 Hamburg, Germany

${ }^{2}$ Universität Hamburg, Luruper Chaussee 149, D-22761 Hamburg, Germany

${ }^{3}$ Ruhr Universität Bochum, Universitätsstr. 150, D-44780 Bochum, Germany

${ }^{4}$ SLAC National Accelerator Laboratory, 2575 Sand Hill Road, Menlo Park, CA 94025, USA

${ }^{5}$ Paul Scherrer Institut (PSI), 5232 Villigen, Switzerland

*Corresponding author: andreas.schropp@desy.de

It was demonstrated in recent experiments that aberration-free nano-focusing of $\mathrm{X}$ rays is possible with a stack of Beryllium compound refractive lenses (Be CRLs) in combination with a corrective phase plate [1,2]. The phase plate operates like an eyeglass rectifying residual errors of the main optics. In this way, diffraction-limited X-ray focusing can be achieved, enhancing the performance of scanning X-ray microscopy based on, e. g., Be CRLs to ultimate limits. We implemented this approach at beamline P06 at PETRA III (DESY, Hamburg) making use of additive manufacturing for phase plate fabrication in order to provide a versatile and easy-to-use nano-focusing platform. In this contribution, we demonstrate the high performance of these optics, which can now produce very clean Gaussian X-ray foci with a size smaller than $100 \mathrm{~nm}$ (FWHM) at moderate working distances larger than $10 \mathrm{~cm}$. This development is especially interesting if larger sample environments are required. For example, in-situ or operando setups often need more infrastructure in the vicinity of a sample to adjust temperature, pressure or gas flow [3].

With a new optical setup at beamline P06 combining the micro- (optics and sample) and nanohutch (diffraction detector), we recently investigated a thin section of a Clunio-larva eye (see G. Falkenberg, et al. [4] and references therein) by scanning X-ray microscopy employing X-ray fluorescence (XRF) and coherent diffraction contrast (ptychography). In this case, a stack of 50 Be CRLs with radius of curvature of $R=50 \mu \mathrm{m}$ and geometric aperture of $D=300 \mu \mathrm{m}$ was used to focus hard $\mathrm{X}$ rays $(E=12 \mathrm{keV})$ to slightly below $100 \mathrm{~nm}(\mathrm{FWHM})$ at a focal length of $f=22 \mathrm{~cm}$.

In a first step the optics were characterized by ptychography using a nano-structured sample having finest lateral structures of $50 \mathrm{~nm}$. It consists of an array of Siemens-stars structured into a $1 \mu \mathrm{m}$ thick tungsten layer on a CVD-diamond substrate. This sample was scanned through the nanofocused X-ray beam over an area of $2 \mu \mathrm{m}$ in horizontal and vertical direction. Far-field diffraction patterns were recorded at each scan point by an Eiger X 4M-detector (Dectris Ltd.), positioned at a distance of $L=6850 \mathrm{~mm}$ behind the sample. From this data set the full information about the nano-focused X-ray beam can be retrieved [5]. By implementing a polymer phase plate the performance could be considerably improved as illustrated in Fig. 1 a), showing a comparison between the X-ray beam caustic with and without the phase plate. Here, the polymer phase plate [cf. inset of Fig. 1 a)] was fabricated using a Nanoscribe 3D-printer. Using this corrective optics the maximum intensity in side lobes surrounding the main focal spot could be decreased to the $1 \%$-level.

Some results of the XRF-experiment on the Clunio-larva eye are summarized in Fig. $1 \mathrm{~b}$ ), demonstrating that we could image an arrangement of pigments with high spatial resolution at a moderate photon flux of $3 \times 10^{9} \mathrm{ph} / \mathrm{s}$. The size of these pigments is typically a few hundred nanometers, 
which can be easily resolved with the new X-ray optics. To date, a similar X-ray imaging performance was only obtained in the P06 nanohutch using nano-focusing lenses made out of silicon. In addition, by switching the pre-focusing lenses in the P06 optics hutch the flux and the coherence length of the X-ray beam at the position of the microscope can be adapted easily depending on experimental needs. For example, in order to illuminate the Be CRLs coherently a stronger pre-focusing was chosen to increase the transverse coherence length of the X-ray beam in the horizontal direction. By matching now the horizontal coherence length to the geometric aperture of the optics, the total photon flux is reduced to about $3 \times 10^{8} \mathrm{ph} / \mathrm{s}$. In this coherent measurement mode diffraction and fluorescence data can be acquired simultaneously.

a)
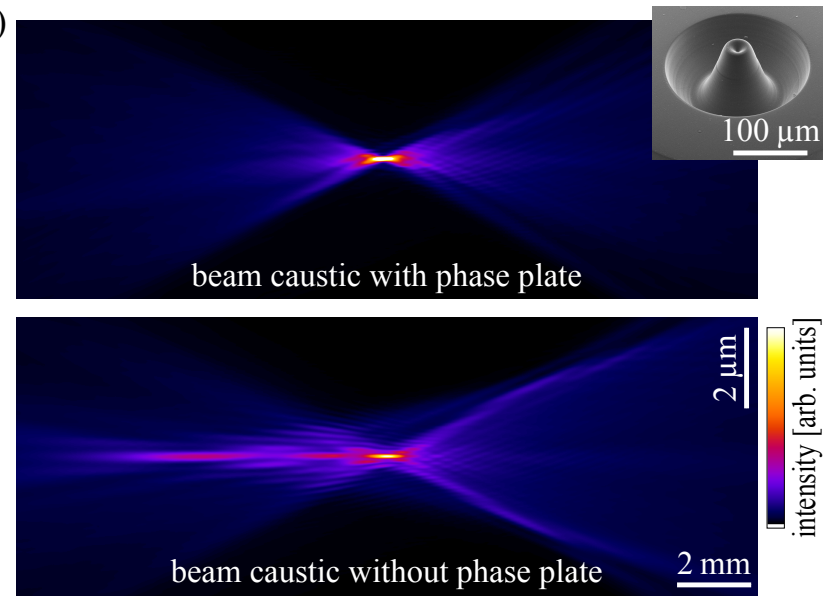

b)
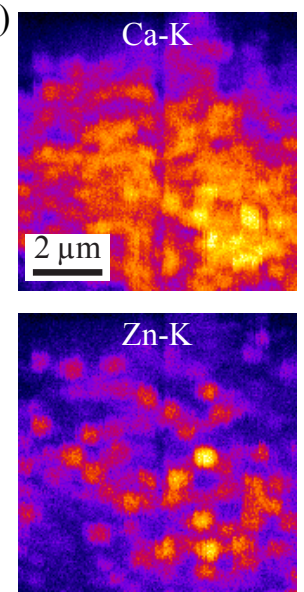
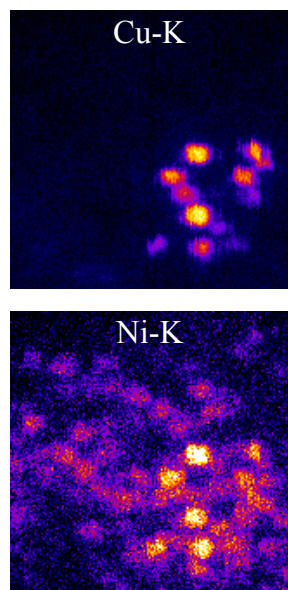

Figure 1: a) Comparison of the beam caustic with and without the phase plate made out of a polymer (see inset). b) Fluorescence maps measured on a thin section of a Clunio-larva eye, showing the elemental distribution of metal-enriched pigments surrounding the photo-sensitive area of the eye.

In conclusion, these new optics developments at beamline P06 promise optimal focusing conditions for multi-modal X-ray imaging [6,7]. Direct scanning microscopy strongly profits from the clean nano-focused X-ray beam and, by using aperture matching schemes, the photon flux through the optics can be optimized either for XRF or ptychographic measurements requiring maximum flux or high spatial coherence, respectively. The transmission of the X-ray optics is high, about $40 \%$ in the presented case, and their large working distance gives one the flexibility to implement complicated and more extended sample environments. Currently available Be CRLs will allow us to reach the $50 \mathrm{~nm}$-resolution regime in direct imaging, and ptychography will further push the spatial resolution to below $10 \mathrm{~nm}$. However, in order to fully exploit the potential of these optics the stability of the setup in the microprobe hutch needs further improvements to finally reach the highest spatial resolution regime [8].

\section{References:}

[1] F. Seiboth, et al., Nat. Comm. 8, 14623 (2017).

[2] F. Seiboth, et al., J. Synchrotron Rad. 25, 108 - 115 (2018).

[3] S. Baier, et al., Microsc. Microanal. 22, 178 - 188 (2016).

[4] G. Falkenberg, et al., J. Phys. Conf. Ser. 463, 012016 (2013).

[5] A. Schropp, et al., Appl. Phys. Lett. 96, 091102 (2010).

[6] A. Schropp, et al., J. Microsc. 241, 9 - 12 (2010).

[7] R. N. Wilke, et al., Geomicrobiol. J. 32, 380 - 393 (2015).

[8] A. Schropp, et al., Appl. Phys. Lett. 100, 253112 (2012). 